\title{
Predicting Spread, Recovery and Death Due to COVID-19 using a Time-Series Model (Prophet)
}

\author{
SK. Golam Mahmud, Mahbub C. Mishu and Dip Nandi
}

\begin{abstract}
The world is facing its biggest challenge since 1920 due to spread of COVID-19 virus. Identified in China in December 2019, the virus has spread more than 200 countries in the world. Scientists have named the virus as Novel Corona Virus (belongs to SARS group virus). The virus has caused severe disruption to our world. Educational institutions, financial Services, government services and many other sectors are badly affected by this virus. More importantly, the virus has caused a massive amount of human deaths around the world and still its infecting people every day. Scientist around the world are trying to find a solution to stop the COVID-19. Their solutions include identifying possible effective vaccine, computeraided modelling to see the pattern of spread etc. Using Machine Learning techniques, it is possible to forecast the spread, death, and recovery due to COVID-19. In this article, we have shown a machine learning model named as Prophet Time Series Analysis to forecast the spread, death, and recovery in different countries. We train the model using the available historical data on COVID19 from John Hopkins University's COVID-19 site. Then we forecast spread, death, and recovery for seven days using a well known forecasting model called Prophet. This interval can be increased to see the effect of COVID-19. We chose 145 days of historical data to train the model then we predict effect for seven days (15 June 2020 to 22 June 2020). To verify out result, we compare the predicted value with actual value of spread, death and recovery. The model provides accuracy over $92 \%$ in all the cases. Our model can be used to identify the effect of COVID-19 in any countries in the world. The system is developed using Python language and visualization is also possible interactively. By using our system, it will be possible to observe the effect of spread, death and recovery for any countries for any period of time.
\end{abstract}

Index Terms-COVID-19, Machine Learning, Prediction, Prophet, Time-Series, Python

\section{INTRODUCTION}

The world has been encountering the global threat of COVID-19 or Coronavirus Disease - 2019 outbreak which began from the Wuhan city of the Hubei province of China during December 2019. Later, the cause of the disease has been identified as SARS-CoV-2, a novel Coronavirus having the capability of human transmission[1], [2]. Figure 1 shows the time-line of COVID-19 [3].

Sk. Golam Mahmud is a student of American International UniversityBangladesh (AIUB). (e-mail: sk_golam_mahmud@yahoo.com).

Mahbub C. Mishu is with American International University-Bangladesh (AIUB). (e-mail: mahbub@aiub.edu).

Dip Nandi is with American International University-Bangladesh (AIUB). (e-mail: dip.nandi@aiub.edu).

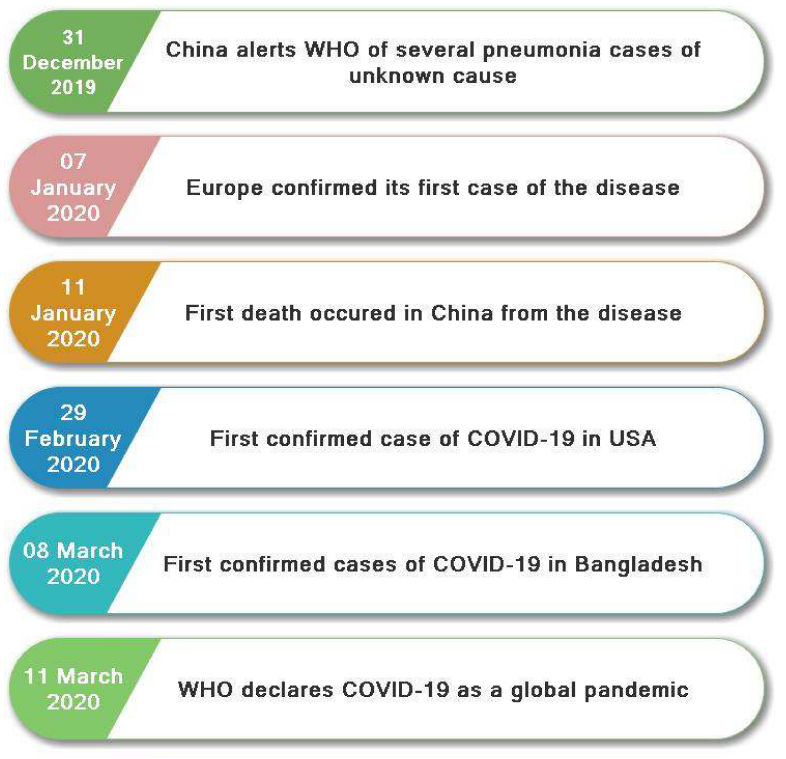

Fig. 1. Time-line of COVID-19 [3]

This global pandemic has also demonstrated that there is no geographical restraint of the disease spread. Responses to COVID-19 in each country depend on the country's resources, influencing the altered case detection rate [4], [5]. The very high number of encounters, high rate of transmission and critical challenges that COVID-19 poses demand the urgency for newer public health initiatives, accurate technical approaches for monitoring and predicting disease course and other preventive measures. Several reports show that many highly populated underdeveloped and developing countries of South Asia, Africa and South America, COVID-19 can be a threat, which can potentially paralyze the health and economic systems [6], [7], [5]. These disastrous effects can be prevented only through adequate and appropriate preparedness. Using a time-series predictive model the disastrous effects can be handled. In this article we have shown the prediction of spread, recovery rate and number of deaths due to COVID-19 disease using the Prophet Forecasting Model.

\section{BACKGROUND \& RELATED WORK}

Discovered in 1966, Coronavirus is a group of enveloped, positive-stranded large RNA viruses that have the potential to infect human and other animals [8]. COVID-19 timeline shows that the World Health Organization (WHO) was alerted by the China Health Authority about cases of pneumonia with unknown origin on 31 December 2019 [8]. Observing the 
danger of this highly contagious disease spread, WHO declared COVID-19 a Public Health Emergency of International Concern (PHEIC) on 30 January, 2020[8], [9]. Respiratory droplets have been identified as the major source of SARSCoV-2 transmission among human contacts, along with fecaloral, aerosol and in few cases, possible vertical transmission [10]. These confirm that any individual of any age and sex can be infected by it. Due to fast spread of COVID-19, many studies have been carried out for prediction of trend and its impact. This section briefs about recent studies which are primarily related to predictive analytics. In Italy, a research conducted by Giulia Giordano et al shows an epidemic prediction model. It compares infected density and the degree of symptoms. A SIDARTHE Model is used by the authors and data from 20 February 2020 (day 1) to 5 April 2020 (day 46) shows how the progressive restrictions, including the most recent lockdown progressively enforced since 9 March 2020, have affected the spread of the epidemic [11], [9]. Also, it shows the effects of social distancing to minimize the spread of the disease. Melanie Bannister et al shows the correlation of temperature and evidence of COVID-19 in Europe. The Study suggest that a higher temperature may reduce the spread of COVID-19. However, the study conflicts with current spread rate of COVID-19 in the higher temperature region [12]. Lucia Russo et al demonstrated a technique to identify the first day of infections and predictions of COVID-19 in Italy. The study was able to estimate that the actual count of confirmed cases of COVID-19 [13]. The author used Susceptible-InfectiousRecovered-Dead (SIDR) model to predict the outbreak at the epicenter three weeks ahead. The trend analysis of COVID19 pandemic in China using globally accepted SIR model developed by Albertine Weber et al [14]. The primary goal of the study showed by Feng Zhang et al is to provide control measures to be considered internationally for global control of this pandemic [15]. The time frame of dataset is from 310 February, 2020 and authors used a time-series model to predict number of confirmed cases and the turning point where the spread is at peak [15]. A probabilistic model proposed by Joel Hellewell et al showed feasibility analysis of controlling the spread of COVID-19. The model considered infections, basic reoccur number, and probability of contacts traced and rate of clinical infections. Results from the study show that, isolation of infected people and contact tracing is not just enough to minimize the rate of spread [16]. Vitaly Volpert et al showed the effect of quarantine model on the spread of virus infection using data analytics. The goal of this work is to present the assessment of placed quarantine mechanism using mathematical modeling [17].

Based on the recent studies on COVID-19 [18], [19], [20], we use a time-series predictive model known as Prophet to predict the spread, recovery rate and number of deaths due to COVID19 in Brazil, USA, Canada, UK, Spain, Italy, Singapore, Japan, China and South Korea.

\section{MAterials AND Method}

In this section, we have shown forecasting of spread, recovery and death due to COVID-19 diseases.The forecasting is done by using a model called Prophet, originally developed by Facebook in 2017. Prophet Forecasting model is known for predicting non-linear time-series data [21]. The trends can be fit with yearly, weekly, and daily seasonality, plus holiday effects [21]. In our research, we have used this forecasting model to predict the spread, recovery and death of COVID19 disease for different countries of the world. We used a decomposable time series model with three main model components: trend, seasonality, and holidays [22], [23]. They are combined in the following equation:

$$
y(t)=g(t)+s(t)+h(t)+\varepsilon(t)
$$

In the above equation, $g(t)$ is piecewise linear or logistic growth curve for modelling non-periodic changes in time series, $s(t)$ is periodic changes (e.g. weekly seasonality), $h(t)$ : effects of holidays with irregular schedules and $\varepsilon(t)$ is error term accounts for any unusual changes not accommodated by the model [24].

Using time as a regressor, Prophet is trying to fit several linear and non linear functions of time as components. We are, in effect, framing the forecasting problem as a curve-fitting exercise rather than looking explicitly at the time-based dependence of each observation within a time series.

Trend: Trend is modelled by fitting a piece wise linear curve over the trend or the non-periodic part of the time series. The linear fitting exercise ensures that it is least affected by spikes/missing data.

$$
g(t)=\left(k+a(t)^{T} \delta\right) t+\left(m+a(t)^{T} \delta\right)
$$

Here, $\mathrm{k}$ is the growth rate; $\delta$ has the rate adjustments and $\mathrm{m}$ is the offset parameter.

To fit and forecast the effects of seasonality, prophet relies on Fourier series to provide a flexible model. Seasonal effects $s(t)$ are approximated by the following function:

$$
\left.s(t)=\sum_{n=1}^{N}\left(a_{n} \cos \left(\frac{2 \pi n t}{P}\right)+b_{n} \sin \left(\frac{2 \pi n t}{P}\right)\right)\right)
$$

$\mathrm{P}$ is the period (365.25 for yearly data and 7 for weekly data) Parameters $a_{1}, b_{1}, \ldots \ldots, a_{N}, b_{N}$ are estimated for a given $\mathrm{N}$ to model seasonality.

Prophet allows the analyst to provide a custom list of past and future events. A window around such days are considered separately and additional parameters are fitted to model the effect of holidays and events. In our research, there is no use of holidays and events.

\section{REsults}

The dataset is collected from GitHub repository published by "John Hopkins University" titled "COVID-19 Data Repository by the Center for Systems Science and Engineering (CSSE) at Johns Hopkins University". From the repository, we have taken three COVID-19 time series datasets for confirmed cases, deaths and recovery for different countries.

These datasets have worldwide date wise data starting from 22 January, 2020. We have collected data up to 14 June, 2020. Then we use the Prophet model to predict next 7 days result 
based on previous data.

From dataset, we have used columns named 'Country/Region' and all above mentioned dates. Therefore each row specifies date wise confirmed/deaths/recovered number of people. We have considered data of total 145 days and analyzed for future predictions. At the same time, we trained other machine learning, time-series and deep-learning models such as Support Vector Machine (SVM), LSTM and ARIMA. The accuracy of SVM was measured as $76.5 \%$; for LSTM accuracy was below $50 \%$ and for ARIMA it was $70.8 \%$. The following equations are used to calculate accuracy, precision and recall score of different models [25].

$$
\text { Accuracy }=\frac{T P+T N}{T P+T N+F P+F N}
$$

where,

- TP: True Positive: Predicted values correctly predicted as actual positive

- FP: Predicted values incorrectly predicted an actual positive. i.e., Negative values predicted as positive

- FN: False Negative: Positive values predicted as negative

- TN: True Negative: Predicted values correctly predicted as an actual negative

$$
\begin{gathered}
\text { Precision }=\frac{T P}{T P+F P} \\
\text { Recall }=\frac{T P}{T P+F N}
\end{gathered}
$$

Table 1 below shows the performance optimization for Prophet, SVM, LSTM and ARIMA.

TABLE I

Performance Optimization of Different Models

\begin{tabular}{|l|l|l|l|l|}
\hline Model & $\begin{array}{l}\text { Precision } \\
(\%)\end{array}$ & $\begin{array}{l}\text { Recall } \\
(\%)\end{array}$ & $\begin{array}{l}\text { Accuracy } \\
(\%)\end{array}$ & $\begin{array}{l}\text { F-Measure } \\
(\%)\end{array}$ \\
\hline Prophet & 89.2 & 0.4 & 93.2 & 3.66 \\
\hline SVM & 68.4 & 4.3 & 76.5 & 8.2 \\
\hline LSTM & 44.6 & 45.9 & 45.32 & 45.8 \\
\hline ARIMA & 58.2 & 62.7 & 70.81 & 60.48 \\
\hline
\end{tabular}

The highest accuracy was achieved by using Prophet Forecasting model. We use this model for spread, recovery and death prediction due to COVID-19 for different countries. The results obtained from the model was further verified by comparing with actual number of cases, recovery and deaths in different countries. 2020. Table 2 and 3 show the calculation of R2 and RMSE (Root Mean Squared Error) score of spread, death and recovery for different countries.

TABLE II

R2 SCORE FOR SPREAD, DEATH AND RECOVERY

\begin{tabular}{|l|l|l|l|}
\hline Country & Spread & Recovery & Death \\
\hline Brazil & 0.9980 & 0.9707 & 0.9998 \\
\hline Canada & 0.9993 & 0.9999 & 0.9994 \\
\hline China & 0.9983 & 0.9999 & 0.9981 \\
\hline Italy & 0.9999 & 0.9994 & 0.9999 \\
\hline Japan & 0.9998 & 0.9917 & 0.9979 \\
\hline Singapore & 0.9995 & 0.9954 & 0.9986 \\
\hline South Korea & 0.9998 & 0.99965 & 0.9998 \\
\hline Spain & 0.9999 & 0.9998 & 0.9995 \\
\hline UK & 0.9996 & 0.9968 & 0.9998 \\
\hline USA & 0.9999 & 0.9983 & 0.9999 \\
\hline
\end{tabular}

TABLE III

Root Mean Squared Error for Spread, Death and Recovery

\begin{tabular}{|l|l|l|l|}
\hline Country & Spread & Recovery & Death \\
\hline Brazil & 115903823.54 & 445864545.44 & 26273.84 \\
\hline Canada & 902833.64 & 22264.29 & 4868.61 \\
\hline China & 938580.02 & 31771.01 & 3949.80 \\
\hline Italy & 509480.90 & 6031080.77 & 4950.47 \\
\hline Japan & 8260.20 & 258851.42 & 259.49 \\
\hline Singapore & 88342.60 & 346991.37 & 0.09 \\
\hline South Korea & 2961.80 & 6204.06 & 1.13 \\
\hline Spain & 988296.94 & 499260.64 & 55128.29 \\
\hline UK & 4025634.49 & 760.03 & 24718.45 \\
\hline USA & 13533542.91 & 51500933.29 & 154675.23 \\
\hline
\end{tabular}

In figure 2-4, we have shown prediction of no. of confirmed cases, deaths and recovery in Brazil. We fit data dated from 22 January 2020 to 14 June 2020 and predicted the confirmed cases from 15 June 2020 to 22 June 2020 (highlighted in red in figures). Then, we compare with actual confirmed cases, deaths and recovery for 7 days (15 June to 22 June) in Brazil. The comparison with actual numbers of confirmed cases in Brazil from 15 June to 22 June shows an accuracy over $90 \%$ using Prophet Forecasting Model.

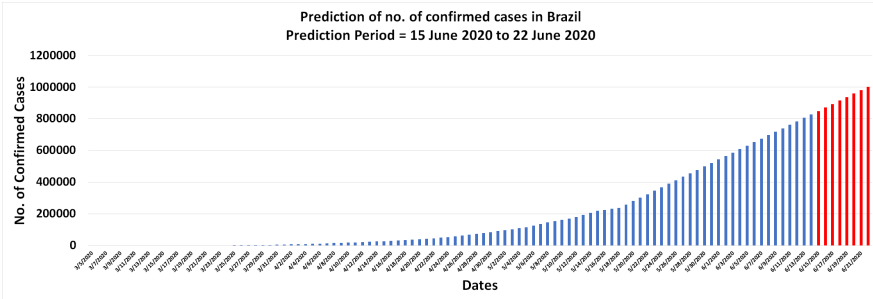

Fig. 2. Prediction of no of Confirmed Cases in Brazil using Prophet Forecasting Model

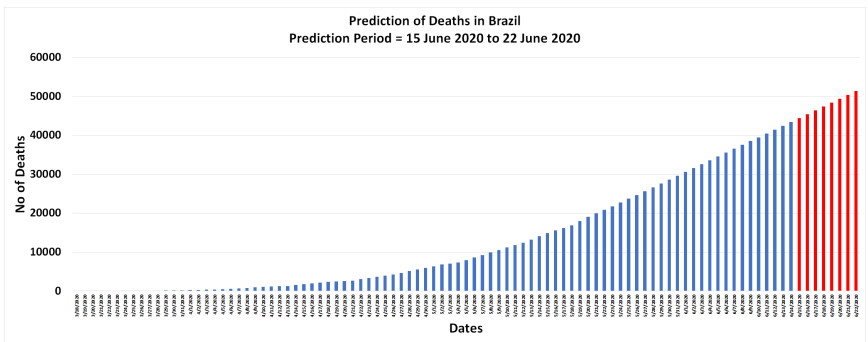

Fig. 3. Prediction of no of Deaths in Brazil using Prophet Forecasting Model

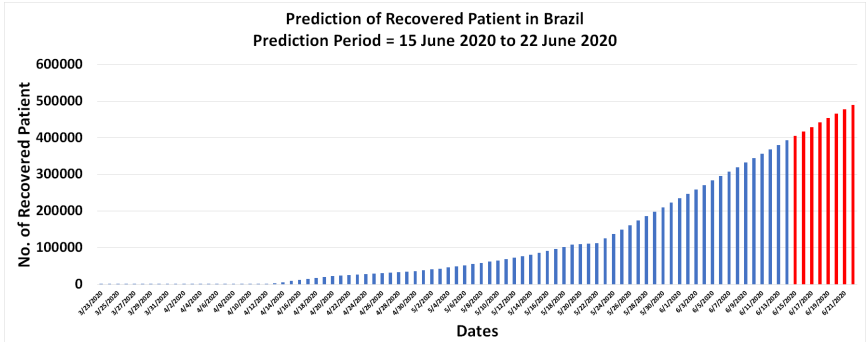

Fig. 4. Prediction of no of Recovery in Brazil using Prophet Forecasting Model

Table 4 shows accuracy of prediction for number of confirmed cases in Brazil for seven days. The average accuracy 
for seven days is calculated as $92.38 \%$. We follow the similar approach to calculate spread, recovery and death for USA, Canada, UK, Spain, Italy, Singapore, Japan, China and South Korea for seven days. These results are summarized in table 7-9 and shown in figure 5-7. Table 5 and 6 shows death and recovery prediction for seven Days in Brazil. The average accuracy of seven days for Death is calculated as $99.38 \%$ and $82.59 \%$ for recovery.

TABLE IV

COMPARISON OF DAILY CONFIRMEd CASES IN BRAZIL

\begin{tabular}{|l|l|l|l|}
\hline Date & Predicted Value & Actual Value & Accuracy \\
\hline $6 / 15 / 2020$ & 849817.86 & 888271 & $95.67 \%$ \\
\hline $6 / 16 / 2020$ & 871691.03 & 923189 & $94.42 \%$ \\
\hline $6 / 17 / 2020$ & 893564.21 & 955377 & $93.53 \%$ \\
\hline $6 / 18 / 2020$ & 915437.38 & 978142 & $93.59 \%$ \\
\hline $6 / 19 / 2020$ & 937310.56 & 1032913 & $90.74 \%$ \\
\hline $6 / 20 / 2020$ & 959183.73 & 1067579 & $89.85 \%$ \\
\hline $6 / 21 / 2020$ & 981056.90 & 1083341 & $90.56 \%$ \\
\hline $6 / 22 / 2020$ & 1002930.08 & 1106470 & $90.64 \%$ \\
\hline
\end{tabular}

TABLE V

Comparison of DaIly Death CASES IN BRAZIL

\begin{tabular}{|l|l|l|l|}
\hline Date & Predicted Value & Actual Value & Accuracy \\
\hline $6 / 15 / 2020$ & 44435.68 & 43959 & $98.93 \%$ \\
\hline $6 / 16 / 2020$ & 45423.29 & 45241 & $99.60 \%$ \\
\hline $6 / 17 / 2020$ & 46410.90 & 46510 & $99.79 \%$ \\
\hline $6 / 18 / 2020$ & 47398.51 & 47748 & $99.27 \%$ \\
\hline $6 / 19 / 2020$ & 48386.13 & 48954 & $98.84 \%$ \\
\hline $6 / 20 / 2020$ & 49373.74 & 49976 & $98.79 \%$ \\
\hline $6 / 21 / 2020$ & 50361.35 & 50591 & $99.55 \%$ \\
\hline $6 / 22 / 2020$ & 51348.97 & 51271 & $99.85 \%$ \\
\hline
\end{tabular}

TABLE VI

COMPARISON OF DAILY RECOVERY CASES IN BRAZIL

\begin{tabular}{|l|l|l|l|}
\hline Date & Predicted Value & Actual Value & Accuracy \\
\hline $6 / 15 / 2020$ & 405053.09 & 477709 & $84.79 \%$ \\
\hline $6 / 16 / 2020$ & 417225.03 & 490005 & $85.15 \%$ \\
\hline $6 / 17 / 2020$ & 429396.97 & 521046 & $82.41 \%$ \\
\hline $6 / 18 / 2020$ & 441568.91 & 534580 & $82.60 \%$ \\
\hline $6 / 19 / 2020$ & 453740.85 & 551631 & $82.25 \%$ \\
\hline $6 / 20 / 2020$ & 465912.79 & 576779 & $80.78 \%$ \\
\hline $6 / 21 / 2020$ & 478084.73 & 588118 & $81.29 \%$ \\
\hline $6 / 22 / 2020$ & 490256.67 & 601736 & $81.47 \%$ \\
\hline
\end{tabular}

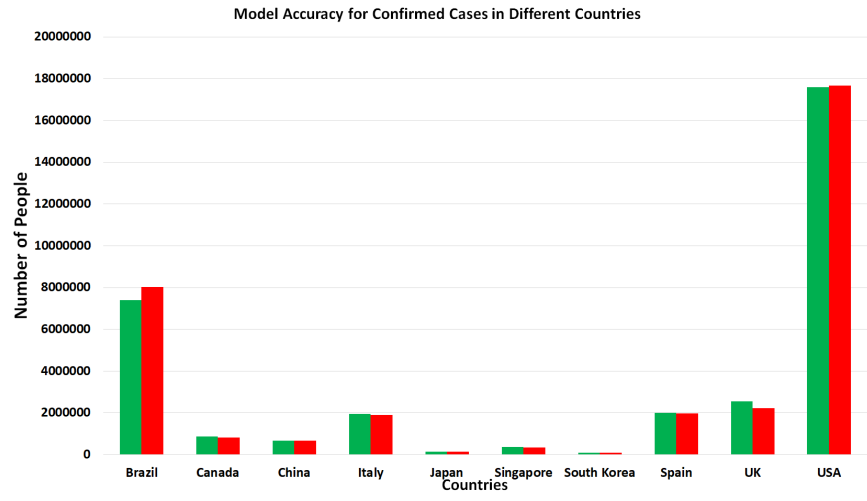

Fig. 5. Model Accuracy for Confirmed Cases in Different Countries

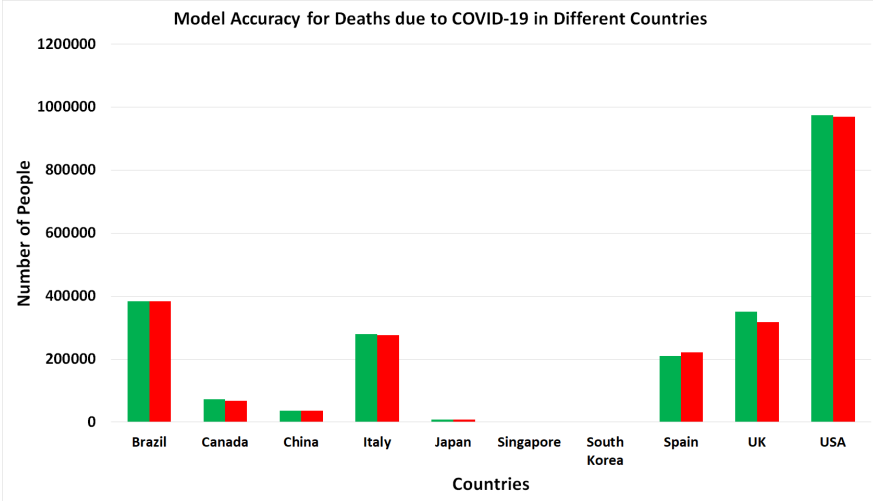

Fig. 6. Model Accuracy for Deaths in Different Countries

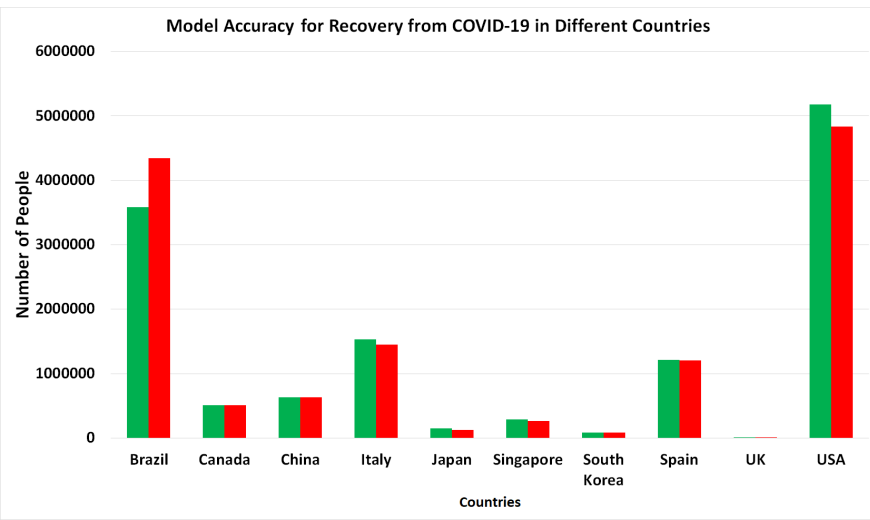

Fig. 7. Model Accuracy for Recovery in Different Countries

TABLE VII

Model ACCuRACy of COVID-19 SpREAD IN DifFERENT Countries

\begin{tabular}{|l|l|l|l|}
\hline \multirow{2}{*}{ Country } & \multicolumn{2}{|l|}{ Confirmed Cases for 7 Days } & \multirow{2}{*}{$\begin{array}{l}\text { Accuracy } \\
\text { in }(\%)\end{array}$} \\
\cline { 2 - 3 } & Predicted Value & Actual Value & 92.23 \\
\hline Brazil & 7410991.79 & 8035282 & 92.23 \\
\hline Canada & 801892.25 & 816734 & 98.18 \\
\hline China & 674075.80 & 675995 & 99.71 \\
\hline Italy & 1879459.60 & 1904282 & 98.70 \\
\hline Japan & 140286.15 & 141024 & 99.47 \\
\hline Singapore & 315475.53 & 332332 & 94.92 \\
\hline South Korea & 96720.19 & 98632 & 98.06 \\
\hline Spain & 1931120.82 & 1962677 & 98.39 \\
\hline UK & 2161658.52 & 2217853 & 97.46 \\
\hline USA & 17601574.44 & 17678123 & 99.56 \\
\hline
\end{tabular}

TABLE VIII

Model Accuracy of COVID-19 Death in DifFerent Countries

\begin{tabular}{|l|l|l|l|}
\hline \multirow{2}{*}{ Country } & \multicolumn{2}{|l|}{ Death Cases for 7 Days } & $\begin{array}{l}\text { Accuracy } \\
(\%)\end{array}$ \\
\cline { 2 - 4 } & Predicted Value & Actual Value & \\
\hline Brazil & 383138.61 & 384250 & 99.71 \\
\hline Canada & 62152.31 & 67022 & 92.73 \\
\hline China & 37106.56 & 37107 & 99.99 \\
\hline Italy & 272289.66 & 276200 & 98.58 \\
\hline Japan & 7398.75 & 7549 & 98.00 \\
\hline Singapore & 206.52 & 208 & 99.28 \\
\hline South Korea & 2225.28 & 2238 & 99.43 \\
\hline Spain & 210173.54 & 221828 & 94.74 \\
\hline UK & 300123.36 & 318299 & 94.28 \\
\hline USA & 964340.44 & 969781 & 99.43 \\
\hline
\end{tabular}


TABLE IX

Model ACCuracy of COVID-19 ReCOVERy In DIFFERENT COUNTRIES

\begin{tabular}{|l|l|l|l|}
\hline \multirow{2}{*}{ Country } & \multicolumn{2}{|l|}{ Recovery Cases for 7 Days } & $\begin{array}{l}\text { Accuracy } \\
(\%)\end{array}$ \\
\cline { 2 - 4 } & Predicted Value & Actual Value & \\
\hline Brazil & 3581239.08 & 4341604 & 82.48 \\
\hline Canada & 507412.89 & 510541 & 99.38 \\
\hline China & 634386.50 & 636138 & 99.72 \\
\hline Italy & 1418952.62 & 1446214 & 98.11 \\
\hline Japan & 122504.29 & 126422 & 96.90 \\
\hline Singapore & 262564.90 & 264394 & 99.30 \\
\hline South Korea & 85931.51 & 86682 & 99.13 \\
\hline Spain & 1195831.32 & 1203008 & 99.40 \\
\hline UK & 10458.66 & 10473 & 99.86 \\
\hline USA & 4675436.06 & 4837649 & 96.64 \\
\hline
\end{tabular}

\section{CONCLUSION}

In this article, we have shown forecast of spread, death and recovery due to COVID-19 using Prophet Time Series Model. The accuracy score shows the effectiveness of our model. By using this model, it is possible to visualize the impact of COVID-19 in any countries. We have also shown a forecasting for seven days but our model can estimate the effect after 15, 30 and 45 days respectively. We have observed the accuracy for different time interval and the accuracy score remains above $90 \%$ in all three cases (Spread, Death and Recovery). Machine Learning techniques are widely used to predict several outcomes such as disease diagnosis, patient's diet, health information etc. These predictions are done by using historical data or survey questionnaires. We have followed the same protocol and used historical data for COVID-19 to train the model. Then we apply the model for forecasting values. Performance optimization of several models have considered and among the models, Prophet's accuracy remain higher.

\section{REFERENCES}

[1] T. P. Velavan and C. G. Meyer, "The covid-19 epidemic," Tropical medicine \& international health, vol. 25, no. 3, p. 278, 2020.

[2] E. Dong, H. Du, and L. Gardner, "An interactive web-based dashboard to track covid-19 in real time," The Lancet infectious diseases, vol. 20, no. 5, pp. 533-534, 2020.

[3] W.-j. Guan, Z.-y. Ni, Y. Hu, W.-h. Liang, C.-q. Ou, J.-x. He, L. Liu, H. Shan, C.-1. Lei, D. S. Hui et al., "Clinical characteristics of 2019 novel coronavirus infection in china," MedRxiv, 2020.

[4] A. B. Massie, B. J. Boyarsky, W. A. Werbel, S. Bae, E. K. Chow, R. K. Avery, C. M. Durand, N. Desai, D. Brennan, J. M. Garonzik-Wang et al., "Identifying scenarios of benefit or harm from kidney transplantation during the covid-19 pandemic: a stochastic simulation and machine learning study," American Journal of Transplantation, vol. 20, no. 11, pp. 2997-3007, 2020.

[5] C. Sáez, N. Romero, J. A. Conejero, and J. M. García-Gómez, "Potential limitations in covid-19 machine learning due to data source variability: A case study in the ncov2019 dataset," Journal of the American Medical Informatics Association, vol. 28, no. 2, pp. 360-364, 2021.

[6] N. Fernandes, "Economic effects of coronavirus outbreak (covid-19) on the world economy," Available at SSRN 3557504, 2020.

[7] M. Buheji, K. da Costa Cunha, G. Beka, B. Mavric, Y. de Souza, S. S. da Costa Silva, M. Hanafi, and T. C. Yein, "The extent of covid-19 pandemic socio-economic impact on global poverty. a global integrative multidisciplinary review," American Journal of Economics, vol. 10, no. 4, pp. 213-224, 2020.

[8] W. H. Organization et al., "Coronavirus disease 2019 (covid-19): situation report, 72," 2020.

[9] R. Dandekar and G. Barbastathis, "Quantifying the effect of quarantine control in covid-19 infectious spread using machine learning," medRxiv, 2020.
[10] P. Mehta, D. F. McAuley, M. Brown, E. Sanchez, R. S. Tattersall, J. J. Manson, H. A. S. Collaboration et al., "Covid-19: consider cytokine storm syndromes and immunosuppression," Lancet (London, England), vol. 395 , no. 10229, p. 1033, 2020.

[11] G. Giordano, F. Blanchini, R. Bruno, P. Colaneri, A. Di Filippo, A. Di Matteo, and M. Colaneri, "Modelling the covid-19 epidemic and implementation of population-wide interventions in italy," Nature Medicine, pp. 1-6, 2020.

[12] M. Bannister-Tyrrell, A. Meyer, C. Faverjon, and A. Cameron, "Preliminary evidence that higher temperatures are associated with lower incidence of covid-19, for cases reported globally up to 29th february 2020," medRxiv, 2020.

[13] C. Anastassopoulou, L. Russo, A. Tsakris, and C. Siettos, "Data-based analysis, modelling and forecasting of the covid-19 outbreak," PloS one, vol. 15, no. 3, p. e0230405, 2020.

[14] A. Weber, F. Ianelli, and S. Goncalves, "Trend analysis of the covid19 pandemic in china and the rest of the world," arXiv preprint arXiv:2003.09032, 2020.

[15] Q. Li, X. Guan, P. Wu, X. Wang, L. Zhou, Y. Tong, R. Ren, K. S. Leung, E. H. Lau, J. Y. Wong et al., "Early transmission dynamics in wuhan, china, of novel coronavirus-infected pneumonia," New England Journal of Medicine, 2020.

[16] J. Hellewell, S. Abbott, A. Gimma, N. I. Bosse, C. I. Jarvis, T. W Russell, J. D. Munday, A. J. Kucharski, W. J. Edmunds, F. Sun et al., "Feasibility of controlling covid-19 outbreaks by isolation of cases and contacts," The Lancet Global Health, 2020.

[17] V. Volpert, M. Banerjee, and S. Petrovskii, "On a quarantine model of coronavirus infection and data analysis," Mathematical Modelling of Natural Phenomena, vol. 15, p. 24, 2020.

[18] S. K. Bandyopadhyay and S. Dutta, "Machine learning approach for confirmation of covid-19 cases: Positive, negative, death and release," medRxiv, 2020.

[19] L. Yan, H.-T. Zhang, J. Goncalves, Y. Xiao, M. Wang, Y. Guo, C. Sun, $\mathrm{X}$. Tang, L. Jin, M. Zhang et al., "A machine learning-based model for survival prediction in patients with severe covid-19 infection," MedRxiv, 2020.

[20] D. Assaf, Y. Gutman, Y. Neuman, G. Segal, S. Amit, S. Gefen-Halevi, N. Shilo, A. Epstein, R. Mor-Cohen, A. Biber et al., "Utilization of machine-learning models to accurately predict the risk for critical covid19," Internal and emergency medicine, vol. 15, no. 8, pp. 1435-1443, 2020.

[21] "Prophet: forecasting at scale," https://research.fb.com/blog/2017/02/prophetforecasting-at-scale/, accessed: 2020-06-12.

[22] S. J. Taylor and B. Letham, "Forecasting at scale," The American Statistician, vol. 72 , no. 1, pp. 37-45, 2018.

[23] M. Navratil and A. Kolkova, "Decomposition and forecasting time series in business economy using prophet forecasting model." Central European Business Review, vol. 8, no. 4, 2019.

[24] E. Ong, M. U. Wong, A. Huffman, and Y. He, "Covid-19 coronavirus vaccine design using reverse vaccinology and machine learning," Frontiers in immunology, vol. 11, p. 1581, 2020.

[25] S. Vaid, C. Cakan, and M. Bhandari, "Using machine learning to estimate unobserved covid-19 infections in north america," The Journal of bone and joint surgery. American volume, 2020.

\section{ACKNOWLEDGMENT}

Authors would like to thank John Hopkins University for research data. Also, authors would like to thank Department of Computer Science, AIUB for providing necessary support to accomplish the research during pandemic. 


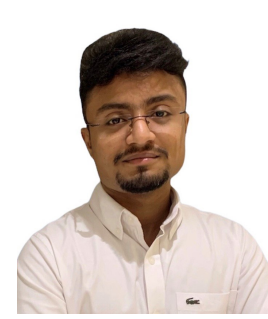

Sk. Golam Mahmud is pursuing his Bachelor degree in Software Engineering program at Department of Computer Science, American International University-Bangladesh. His primary research interests include Data Science, Machine Learning and Computer Vision.

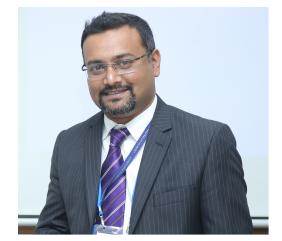

Mahbub C. Mishu received his $\mathrm{PhD}$ from Bournemouth University, UK in 2015 and MSc from the University of Nottingham, UK in 2010. His research interests are mainly focused on Intelligent System Design, Machine Learning and Big Data Analytics. In his Doctoral research, he developed a prototype of Pressure Ulcer Detection and Prevention System based on human physiological parameters and surface (mattress) properties. The system was fully intelligent and capable of providing real-time prevention for Pressure Ulcer Occurrence in human body. After his PhD, he joined Northern Regional College, UK as a Lecturer. He also obtained Higher Education Teaching and Learning Qualification from Ulster University, UK in 2017. He has started working as an Assistant Professor at American International University-Bangladesh since January 2018. Dr Mishu has published several articles in local and international peer reviewed journals and conferences.

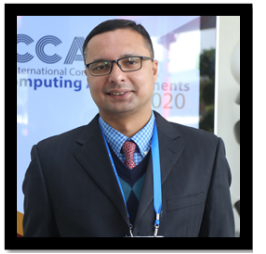

Dip Nandi currently works as an Associate Professor and the Director of Faculty of Science and Technology in American International University-Bangladesh (AIUB). In past, he was the Head of Computer Science Department. Dr. Nandi achieved his Doctor of Philosophy (PhD) degree from RMIT, Australia and MSc degree from The University of Melbourne, Australia. His research area includes: Software Engineering, ELearning Technologies, Data Mining and Information systems. He has supervised more than 70 students as thesis supervisor. Dr. Nandi is associated with several organization such as IEEE, ACM. He has published several peer-reviewed journal articles. Dr. Nandi is a member of Academic Council of AIUB. 